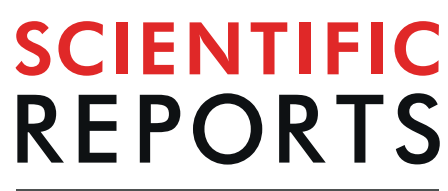

natureresearch

\title{
OPEN Breastfeeding and maternal cardiovascular risk factors: 1982 Pelotas Birth Cohort
}

Received: 6 April 2018

Accepted: 23 August 2019

Published online: 11 September 2019

\author{
Natália P. Lima $\mathbb{D}^{1}{ }^{1}$, Diego G. Bassani $\mathbb{1}^{2,3}$, Elma Izze S. Magalhães ${ }^{1}$, Fernando C. Barros ${ }^{4} \&$ \\ Bernardo L. Horta $\mathbb{D}^{1}$
}

This study evaluated the association of breastfeeding duration with maternal metabolic cardiovascular risk factors among women who have been prospectively followed since birth in a southern Brazilian city. In the unadjusted analysis, total cholesterol was higher among women who never breastfed in relation to those who breastfed $\geq \mathbf{1 2}$ months. Among women with one livebirth, a shorter duration of breastfeeding was associated with lower $\mathrm{HDL}$, while those with two or more livebirths and that breastfed for shorter time presented lower pulse wave velocity, glycaemia and non-HDL measures. After controlling for confounding variables, the magnitude of these associations decreased, and the confidence intervals included the reference. Concerning the duration of breastfeeding of the last child, the analysis was stratified by time since last birth. After controlling for confounders, systolic blood pressure was lower among women who breastfed 3 to $<6$ months and had a child within the last five years in relation to those who breastfed $\geq 6$, but no clear trend was observed $(p=0.17)$. In conclusion, our findings suggest that there is no association between lactation and maternal cardiometabolic risk factors.

Beyond the known short ${ }^{1}$ and long-term benefits ${ }^{2,3}$ for the breastfed children, it has been reported that breastfeeding would be associated with maternal health, women who breastfeed have lower risk of breast cancer and higher birth spacing due to lactational amenorrhea ${ }^{4}$. It has been estimated that breastfeeding prevents about 20,000 deaths from breast cancer every year ${ }^{5}$. Additionally, it has been shown that long duration of breastfeeding reduces the risk of coronary heart disease $e^{6,7}$, type 2 diabetes ${ }^{4,8-10}$, whereas the evidence supporting the association with blood pressure $\mathrm{e}^{11-22}$ and lipid profile $\mathrm{e}^{12,16,18,19,22,23}$ are not clear.

Most of the studies on the long-term consequences of breastfeeding on maternal health have been carried out in high-income countries, where breastfeeding is positively associated with socioeconomic status ${ }^{5}$. Because cardiovascular risk factors are also associated with socioeconomic status ${ }^{24-27}$ and most studies adjusted the estimates for few socioeconomic confounders (e.g. only for schooling), perhaps not capturing its entire dimension, the possibility of residual confounding by socioeconomic status must be considered.

In the present study, we aimed at assessing the association of breastfeeding duration with maternal metabolic risk factors for cardiovascular disease among parous women who have been prospectively followed since birth in a southern Brazilian city, a setting where no strong social patterning of breastfeeding exists.

\section{Results}

In 2012-13, when the participants were 29-31 years of age, we interviewed 1914 of the 2876 women born in 1982, which after taking into account the deaths identified among the cohort members, represented a follow-up rate of $71.0 \%$. And 1147 had delivered at least one live birth and were not pregnant when interviewed, meeting the eligibility criteria. Information on breastfeeding duration and at least one of the metabolic cardiovascular risk factors was available for 1136 of the women eligible to enter the study.

\footnotetext{
${ }^{1}$ Postgraduate Program in Epidemiology, Universidade Federal de Pelotas, Pelotas, Brazil. ${ }^{2}$ Centre for Global Child Health, The Hospital for Sick Children, Toronto, Canada. ${ }^{3}$ Department of Paediatrics and Dalla Lana School of Public Health, University of Toronto, Toronto, Canada. ${ }^{4}$ Postgraduate Program in Health and Behavior, Universidade Católica de Pelotas, Pelotas, Rio Grande do Sul, Brazil. Correspondence and requests for materials should be addressed to N.P.L. (email: natyplima@hotmail.com)
} 


\begin{tabular}{|c|c|c|c|}
\hline Characteristics & $\mathbf{N}$ & $\%$ & Mean (SD) \\
\hline \multicolumn{4}{|l|}{ Skin color } \\
\hline White & 837 & 73.7 & - \\
\hline Black & 197 & 17.3 & \\
\hline Brown/Indigenous/Asian & 102 & 9.0 & \\
\hline European ancestry & 970 & - & $0.75 \pm 0.20$ \\
\hline \multicolumn{4}{|l|}{ Asset index ${ }^{Y}$} \\
\hline $\mathrm{D} / \mathrm{E}$ (poorest) & 57 & 6.4 & - \\
\hline $\mathrm{C}$ & 334 & 37.5 & - \\
\hline $\mathrm{A} / \mathrm{B}$ (richest) & 499 & 56.1 & - \\
\hline Years of schooling & 1134 & - & $10.29 \pm 3.96$ \\
\hline \multicolumn{4}{|l|}{ Total duration of breastfeeding (months)* } \\
\hline$<1$ & 124 & 10.9 & - \\
\hline $1-<3$ & 88 & 7.8 & - \\
\hline $3-<6$ & 142 & 12.5 & - \\
\hline $6-<12$ & 263 & 23.2 & - \\
\hline$\geq 12$ & 519 & 45.6 & - \\
\hline \multicolumn{4}{|l|}{ Parity } \\
\hline 1 & 599 & 52.7 & - \\
\hline 2 & 336 & 29.6 & - \\
\hline$\geq 3$ & 201 & 17.7 & - \\
\hline Systolic blood pressure $(\mathrm{mmHg})$ & 1133 & - & $114.17 \pm 12.13$ \\
\hline Diastolic blood pressure (mmHg) & 1133 & - & $73.44 \pm 9.14$ \\
\hline Carotid intima-media thickness $(\mu \mathrm{m})$ & 983 & - & $580.40 \pm 16.22$ \\
\hline Pulse wave velocity $(\mathrm{m} / \mathrm{s})$ & 496 & - & $6.41 \pm 1.05$ \\
\hline Glycaemia (mg/dl) & 1122 & - & $87.34 \pm 22.91$ \\
\hline Cholesterol (mg/dl) & 1122 & - & $187.12 \pm 35.39$ \\
\hline $\mathrm{LDL}(\mathrm{mg} / \mathrm{dl})$ & 1122 & - & $107.03 \pm 28.49$ \\
\hline $\mathrm{HDL}(\mathrm{mg} / \mathrm{dl})$ & 1122 & - & $60.53 \pm 12.74$ \\
\hline Non-HDL (mg/dl) & 1122 & - & $126.59 \pm 32.83$ \\
\hline Triglycerides $(\mathrm{mg} / \mathrm{dl})^{\#}$ & 1122 & - & $90.13 \pm 1.65$ \\
\hline
\end{tabular}

Table 1. Biological, socioeconomic, behavioral and health characteristics of the studied population $(n=1136)$. Pelotas, 2012. ${ }^{¥}$ Brazilian Association of Research Companies. *Accumulated months of breastfeeding across all pregnancies resulting in $\geq 1$ livebirth. ${ }^{\#}$ Geometric mean.

Table 1 shows that $73.7 \%$ of the women included in the present study were white, mean proportion of European ancestry was $75 \%$, and mean achieved schooling was 10.3 years. Over half of the women (52.7\%) were primiparous and $45.6 \%$ had breastfed for at least 12 months (total number of months breastfeeding).

Regarding the confounding variables, breastfeeding was higher in women with lower socioeconomic status in 2004-5. Systolic and diastolic blood pressure were negatively associated with family income in $2004-5$, whereas carotid intima-media thickness was inversely associated with schooling (Supplementary Table S1). HDL cholesterol was directly associated with socioeconomic status (Supplementary Table S2).

The association between total duration of breastfeeding and blood pressure measures, carotid intima media-thickness and pulse wave velocity is presented in Table 2 . In the analysis stratified by parity, in the crude analysis, women who breastfed 3 to $<6$ months and had had two or more livebirths showed lower pulse wave velocity than those who breastfed $\geq 12$ months $(\beta=-0.79,95 \% \mathrm{CI}:-1.4 ;-0.2, p=0.04)$, but there was no clear pattern of association. After adjustment for the confounders, the magnitude of the estimative barely changed, but the confidence interval included the reference $(\beta=-0.80,95 \% \mathrm{CI}:-1.7 ; 0.1$, p-trend $=0.23)$. Blood pressure measures and carotid intima-media thickness did not present an association with breastfeeding. Additionally, no interaction between parity and breastfeeding was verified.

Table 3 shows the association between total duration of breastfeeding and glycaemia and blood lipids. In the unadjusted analysis, total cholesterol was higher among women who never breastfed when comparing with those who breastfed for 12 months or longer $(\beta=7.17,95 \%$ CI: $0.2 ; 14.2)$, but we did not observe a clear trend toward increasing total cholesterol, as duration of breastfeeding decreased ( $p$-trend $=0.13$ ). Furthermore, after controlling for confounders, the regression coefficient among those who never breastfed decreased from 7.17 to 2.27 (95\% CI: - 6.8; 11.3). Glycaemia and cholesterol fractions were not associated with breastfeeding and the magnitude of the regression coefficients decreased after controlling for confounding variables. On the other hand, when the analyses were stratified for parity, in the crude analysis, women who had had one livebirth and breastfed for $3-<6$ showed lower HDL than those who breastfed $\geq 12$ months $(\beta=-3.16 ; 95 \% \mathrm{CI}:-6.1 ;-0.2)$, although no linear association was observed $(\mathrm{p}=0.11)$. But in the adjusted analysis, the regression coefficient reduced to $-0.44(95 \% \mathrm{CI}:-4.1 ; 3.2)$. Among those women who had had two or more livebirths, glycaemia and non-HDL 


\begin{tabular}{|c|c|c|c|c|c|c|c|c|c|}
\hline & \multirow[b]{3}{*}{$\mathbf{N}$} & \multicolumn{8}{|c|}{ Regression coefficient ( $95 \%$ confidence interval) } \\
\hline & & \multicolumn{2}{|c|}{$\begin{array}{l}\begin{array}{l}\text { Systolic blood pressure } \\
(\mathrm{mmHg})\end{array} \\
\end{array}$} & \multicolumn{2}{|c|}{$\begin{array}{l}\text { Diastolic blood pressure } \\
(\mathrm{mmHg})\end{array}$} & \multicolumn{2}{|c|}{$\begin{array}{l}\text { Intima-media thickness } \\
(\mu \mathrm{m})\end{array}$} & \multicolumn{2}{|c|}{ Pulse wave velocity $(\mathrm{m} / \mathrm{s})$} \\
\hline & & Crude & Adjusted $^{\dagger Y}$ & Crude & Adjusted $^{\dagger Y}$ & Crude & Adjusted $^{\dagger}$ & Crude & Adjusted $^{\dagger}$ \\
\hline Breastfeeding & & $\mathrm{p}=0.66^{*}$ & $\mathrm{p}=0.97 *$ & $\mathrm{p}=0.36^{*}$ & $\mathrm{p}=0.80^{*}$ & $\mathrm{p}=0.40^{*}$ & $\mathrm{p}=0.80^{*}$ & $\mathrm{p}=0.28^{*}$ & $\mathrm{p}=0.71 *$ \\
\hline Never & 124 & \begin{tabular}{|l}
0.51 \\
$(-1.8 ; 2.8)$ \\
\end{tabular} & $\begin{array}{l}-0.05 \\
(-2.8 ; 2.7) \\
\end{array}$ & $\begin{array}{l}0.80 \\
(-0.9 ; 2.5) \\
\end{array}$ & \begin{tabular}{|l}
0.27 \\
$(-1.8 ; 2.3)$ \\
\end{tabular} & \begin{tabular}{|l|}
1.41 \\
$(-1.9 ; 4.7)$ \\
\end{tabular} & $\begin{array}{l}-0.51 \\
(-4.4 ; 3.4) \\
\end{array}$ & \begin{tabular}{|l|}
0.16 \\
$(-0.1 ; 0.5)$ \\
\end{tabular} & $\begin{array}{l}0.07 \\
(-0.3 ; 0.5)\end{array}$ \\
\hline Ever & 1012 & $\operatorname{Ref}(0)$ & $\operatorname{Ref}(0)$ & $\operatorname{Ref}(0)$ & $\operatorname{Ref}(0)$ & $\operatorname{Ref}(0)$ & $\operatorname{Ref}(0)$ & $\operatorname{Ref}(0)$ & $\operatorname{Ref}(0)$ \\
\hline Total sum of breastfeeding & & $\mathrm{p}=0.96^{* *}$ & $\mathrm{p}=0.81^{* *}$ & $\mathrm{p}=0.73^{* *}$ & $\mathrm{p}=0.90^{*}$ & $\mathrm{p}=0.82 * *$ & $\mathrm{P}=0.43^{*}$ & $\mathrm{p}=0.33^{*}$ & $\mathrm{p}=0.69^{*}$ \\
\hline Never & 124 & $\begin{array}{l}0.28 \\
(-2.1 ; 2.7)\end{array}$ & $\begin{array}{l}-0.28 \\
(-3.2 ; 2.7)\end{array}$ & $\begin{array}{l}0.64 \\
(-1.2 ; 2.4)\end{array}$ & $\begin{array}{l}0.10 \\
(-2.0 ; 2.2)\end{array}$ & $\begin{array}{l}1.23 \\
(-2.2 ; 4.7)\end{array}$ & $\begin{array}{l}-0.81 \\
(-4.9 ; 3.3)\end{array}$ & $\begin{array}{l}0.16 \\
(-0.2 ; 0.5)\end{array}$ & $\begin{array}{l}0.04 \\
(-0.4 ; 0.5)\end{array}$ \\
\hline $1-<3$ & 88 & $\begin{array}{l}-0.41 \\
(-3.2 ; 2.3)\end{array}$ & $\begin{array}{l}-0.17 \\
(-4.0 ; 3.7)\end{array}$ & $\begin{array}{l}-0.77 \\
(-2.8 ; 1.3)\end{array}$ & $\begin{array}{l}-0.71 \\
(-3.5 ; 2.1)\end{array}$ & $\begin{array}{l}-0.67 \\
(-4.7 ; 3.3)\end{array}$ & $\begin{array}{l}3.64 \\
(-1.7 ; 9.0)\end{array}$ & $\begin{array}{l}-0.06 \\
(-0.4 ; 0.3)\end{array}$ & $\begin{array}{l}-0.02 \\
(-0.6 ; 0.5)\end{array}$ \\
\hline $3-<6$ & 142 & $\begin{array}{l}-0.65 \\
(-2.9 ; 1.6)\end{array}$ & $\begin{array}{l}-0.50 \\
(-3.3 ; 2.2)\end{array}$ & $\begin{array}{l}0.21 \\
(-1.5 ; 1.9)\end{array}$ & $\begin{array}{l}0.28 \\
(-1.7 ; 2.3)\end{array}$ & $\begin{array}{l}-1.08 \\
(-4.3 ; 2.2)\end{array}$ & $\begin{array}{l}-0.73 \\
(-4.5 ; 3.0)\end{array}$ & $\begin{array}{l}-0.20 \\
(-0.5 ; 0.1)\end{array}$ & $\begin{array}{l}-0.24 \\
(-0.6 ; 0.1)\end{array}$ \\
\hline $6-<12$ & 263 & $\begin{array}{l}-0.40 \\
(-2.2 ; 1.4)\end{array}$ & $\begin{array}{l}-0.53 \\
(-2.8 ; 1.8)\end{array}$ & $\begin{array}{l}-0.48 \\
(-1.8 ; 0.9)\end{array}$ & $\begin{array}{l}-0.64 \\
(-2.3 ; 1.0)\end{array}$ & $\begin{array}{l}0.13 \\
(-2.5 ; 2.7)\end{array}$ & $\begin{array}{l}-1.72 \\
(-4.9 ; 1.5)\end{array}$ & $\begin{array}{l}0.11 \\
(-0.1 ; 0.4)\end{array}$ & $\begin{array}{l}0.06 \\
(-0.3 ; 0.4)\end{array}$ \\
\hline$\geq 12$ & 519 & $\operatorname{Ref}(0)$ & $\operatorname{Ref}(0)$ & $\operatorname{Ref}(0)$ & $\operatorname{Ref}(0)$ & $\operatorname{Ref}(0)$ & $\operatorname{Ref}(0)$ & $\operatorname{Ref}(0)$ & $\operatorname{Ref}(0)$ \\
\hline Parity $=1$ & & \multicolumn{2}{|l|}{$\mathrm{P}=0.91^{*}$} & \multicolumn{2}{|l|}{$\mathrm{P}=0.95^{\ddagger}$} & \multicolumn{2}{|l|}{$\mathrm{P}=0.42^{\ddagger}$} & \multicolumn{2}{|l|}{$\mathrm{P}=0.70^{\ddagger}$} \\
\hline Total sum of breastfeeding & & $\mathrm{P}=0.41^{*}$ & $\mathrm{p}=0.68^{*}$ & $\mathrm{P}=0.36^{*}$ & $\mathrm{p}=0.64^{* *}$ & $\mathrm{P}=0.08^{* *}$ & $\mathrm{P}=0.47^{* *}$ & $\mathrm{P}=0.12 * *$ & $\mathrm{P}=0.28^{* *}$ \\
\hline Never & 97 & $\begin{array}{l}1.52 \\
(-1.2 ; 4.2) \\
\end{array}$ & $\begin{array}{l}0.11 \\
(-3.1 ; 3.4) \\
\end{array}$ & \begin{tabular}{|l|}
0.92 \\
$(-1.3 ; 3.1)$ \\
\end{tabular} & $\begin{array}{l}-0.19 \\
(-2.7 ; 2.4) \\
\end{array}$ & $\begin{array}{l}3.40 \\
(-0.6 ; 7.4) \\
\end{array}$ & \begin{tabular}{|l|}
0.79 \\
$(-4.2 ; 5.8)$ \\
\end{tabular} & \begin{tabular}{|l|}
0.35 \\
$(-0.1 ; 0.7)$ \\
\end{tabular} & \begin{tabular}{|l|}
0.28 \\
$(-0.2 ; 0.7)$ \\
\end{tabular} \\
\hline $1-<3$ & 71 & $\begin{array}{l}-1.65 \\
(-4.7 ; 1.4)\end{array}$ & $\begin{array}{l}-1.52 \\
(-5.4 ; 2.4)\end{array}$ & $\begin{array}{l}-1.83 \\
(-4.3 ; 0.6)\end{array}$ & $\begin{array}{l}-1.53 \\
(-4.6 ; 1.5)\end{array}$ & $\begin{array}{l}1.36 \\
(-3.1 ; 5.8)\end{array}$ & $\begin{array}{l}3.89 \\
(-2.2 ; 9.9)\end{array}$ & $\begin{array}{l}0.24 \\
(-0.2 ; 0.7)\end{array}$ & $\begin{array}{l}0.24 \\
(-0.4 ; 0.8)\end{array}$ \\
\hline $3-<6$ & 108 & $\begin{array}{l}-0.71 \\
(-3.3 ; 1.9)\end{array}$ & $\begin{array}{l}-1.94 \\
(-5.0 ; 1.1)\end{array}$ & $\begin{array}{l}-0.50 \\
(-2.6 ; 1.6)\end{array}$ & $\begin{array}{l}-1.10 \\
(-3.5 ; 1.3)\end{array}$ & $\begin{array}{l}-0.27 \\
(-4.1 ; 3.5)\end{array}$ & $\begin{array}{l}-1.46 \\
(-6.1 ; 3.1)\end{array}$ & $\begin{array}{l}0.21 \\
(-0.2 ; 0.6)\end{array}$ & $\begin{array}{l}0.08 \\
(-0.4 ; 0.5)\end{array}$ \\
\hline $6-<12$ & 152 & $\begin{array}{l}-0.18 \\
(-2.6 ; 2.2)\end{array}$ & $\begin{array}{l}-0.20 \\
(-3.0 ; 2.6)\end{array}$ & $\begin{array}{l}-0.55 \\
(-2.5 ; 1.4)\end{array}$ & $\begin{array}{l}-0.46 \\
(-2.7 ; 1.7)\end{array}$ & $\begin{array}{l}-0.71 \\
(-4.1 ; 2.7)\end{array}$ & $\begin{array}{l}-0.96 \\
(-5.2 ; 3.3)\end{array}$ & $\begin{array}{l}0.33 \\
(-0.1 ; 0.7)\end{array}$ & $\begin{array}{l}0.24 \\
(-0.2 ; 0.6)\end{array}$ \\
\hline$\geq 12$ & 171 & $\operatorname{Ref}(0)$ & $\operatorname{Ref}(0)$ & $\operatorname{Ref}(0)$ & $\operatorname{Ref}(0)$ & $\operatorname{Ref}(0)$ & $\operatorname{Ref}(0)$ & $\operatorname{Ref}(0)$ & $\operatorname{Ref}(0)$ \\
\hline \multicolumn{10}{|l|}{ Parity $>1$} \\
\hline Total sum of breastfeeding & & $\mathrm{P}=0.25^{*}$ & $\mathrm{p}=0.21^{* *}$ & $\mathrm{P}=0.56^{*}$ & $\mathrm{p}=0.34^{* *}$ & $\mathrm{P}=0.48^{*}$ & $\mathrm{P}=0.41^{* *}$ & $\mathrm{P}=0.04^{*}$ & $\mathrm{P}=0.23 * *$ \\
\hline Never & 27 & $\begin{array}{l}-4.92 \\
(-10.2 ; 0.3)\end{array}$ & $\begin{array}{l}-4.05 \\
(-10.6 ; 2.5)\end{array}$ & $\begin{array}{l}-1.76 \\
(-5.5 ; 2.0)\end{array}$ & $\begin{array}{l}-1.95 \\
(-6.4 ; 2.5)\end{array}$ & $\begin{array}{l}-2.32 \\
(-9.8 ; 5.2)\end{array}$ & $\begin{array}{l}-4.92 \\
(-13.7 ; 3.8)\end{array}$ & $\begin{array}{l}0.55 \\
(-0.3 ; 1.4)\end{array}$ & $\begin{array}{l}-0.27 \\
(-1.5 ; 1.0)\end{array}$ \\
\hline $1-<3$ & 17 & $\begin{array}{l}3.94 \\
(-2.6 ; 10.5) \\
\end{array}$ & $\begin{array}{l}-2.78 \\
(-13.9 ; 8.3) \\
\end{array}$ & $\begin{array}{l}2.00 \\
(-2.6 ; 6.6)\end{array}$ & $\begin{array}{l}-3.33 \\
(-11.0 ; 4.3) \\
\end{array}$ & $\begin{array}{l}-4.22 \\
(-13.7 ; 5.3)\end{array}$ & $\begin{array}{l}-1.01 \\
(-16.9 ; 14.9)\end{array}$ & $\begin{array}{l}-0.39 \\
(-1.6 ; 0.8) \\
\end{array}$ & $\begin{array}{l}-0.35 \\
(-2.8 ; 2.1)\end{array}$ \\
\hline $3-<6$ & 34 & $\begin{array}{l}-1.08 \\
(-5.9 ; 3.7) \\
\end{array}$ & $\begin{array}{l}0.19 \\
(-5.9 ; 6.3) \\
\end{array}$ & $\begin{array}{l}1.31 \\
(-2.1 ; 4.7)\end{array}$ & $\begin{array}{l}1.44 \\
(-2.8 ; 5.6) \\
\end{array}$ & $\begin{array}{l}0.24 \\
(-6.7 ; 7.2) \\
\end{array}$ & $\begin{array}{l}3.46 \\
(-4.7 ; 11.6) \\
\end{array}$ & $\begin{array}{l}-0.79 \\
(-1.4 ;-0.2)\end{array}$ & $\begin{array}{l}-0.80 \\
(-1.7 ; 0.1)\end{array}$ \\
\hline $6-<12$ & 111 & $\begin{array}{l}-0.90 \\
(-3.8 ; 2.0)\end{array}$ & $\begin{array}{l}-1.89 \\
(-5.9 ; 2.1) \\
\end{array}$ & $\begin{array}{l}-0.77 \\
(-2.8 ; 1.3) \\
\end{array}$ & $\begin{array}{l}-1.78 \\
(-4.5 ; 0.9) \\
\end{array}$ & \begin{tabular}{|l|}
2.98 \\
$(-1.3 ; 7.2)$ \\
\end{tabular} & $\begin{array}{l}-2.33 \\
(-7.5 ; 2.9)\end{array}$ & \begin{tabular}{|l|}
0.07 \\
$(-0.3 ; 0.4)$ \\
\end{tabular} & \begin{tabular}{|l}
0.04 \\
$(-0.6 ; 0.7)$
\end{tabular} \\
\hline$\geq 12$ & 348 & $\operatorname{Ref}(0)$ & $\operatorname{Ref}(0)$ & $\operatorname{Ref}(0)$ & $\operatorname{Ref}(0)$ & $\operatorname{Ref}(0)$ & $\operatorname{Ref}(0)$ & $\operatorname{Ref}(0)$ & $\operatorname{Ref}(0)$ \\
\hline
\end{tabular}

Table 2. Blood pressure, carotid intima-media thickness and pulse wave velocity according to total breastfeeding $(\mathrm{n}=1136)$. Pelotas, $2012 .^{\dagger}$ Adjusted for genomic ancestry, family income and maternal schooling at birth; asset index at childhood; and family income, schooling, asset index, energy intake, physical activity, alcohol consumption, current smoking and BMI at 2004-5. ${ }^{¥}$ Systolic and diastolic blood pressure were also adjusted for use of hypertensive drugs. *Test for heterogeneity. ${ }^{*}$ Test for linear trend. ${ }^{*} \mathrm{p}$-value for interaction.

were lower in women with shorter duration of breastfeeding, than in those who breastfed 12 months or longer, but the associations did not show a clear pattern. After adjustment, glycaemia was lower among women who breastfed for 6 to $<12$ months $(\beta=-5.47,95 \% \mathrm{CI}:-10.4 ;-0.5, \mathrm{p}=0.15)$, whereas for non-HDL, the crude analysis shown lower measure among those with 1 to $<3$ months of breastfeeding $(\beta=-17.3,95 \% \mathrm{CI}:-33.5 ;-1.2)$, but the confidence interval included the reference after adjustment and no association was observed $(\mathrm{p}=0.12)$. Also, there was no significant interaction between breastfeeding and parity.

Tables 4 and 5 show the results of the analyses restricted to duration of breastfeeding of the last child, after adjusting for confounders. These analyses were stratified for time since last birth. Systolic blood pressure was lower among women who breastfed 3 to $<6$ months and had a child within the last five years when compared with those who breastfed $\geq 6(\beta=-3.36,95 \% \mathrm{CI}:-6.7 ;-0.5)$, though no clear trend was observed $(\mathrm{p}=0.17)$. We did not observe association in those whose last childbirth was $\geq 5$ years and there was no interaction ( $\mathrm{p}$-interaction $=0.13$ ). Regarding the other cardiovascular risk factors, we did not observe association with the last child breastfeeding.

\section{Discussion}

In a population that has been prospectively followed since birth, after controlling for biological, socioeconomic and behavioral variables, no association between breastfeeding and maternal cardiometabolic risk factors was observed.

Observational studies evaluating the association of breastfeeding with maternal health outcomes have reported benefits on metabolic risk factors, such as blood pressure ${ }^{11,13-16,18-22}$, glucose $e^{9,10,16,19,21,22,28-30}$ and 


\begin{tabular}{|c|c|c|c|c|c|c|c|c|c|c|c|c|c|}
\hline & \multirow[b]{3}{*}{$\mathbf{N}$} & \multicolumn{12}{|c|}{ Regression coefficient ( $95 \%$ confidence interval) } \\
\hline & & \multicolumn{2}{|c|}{ Glycaemia (mg/dl) } & \multicolumn{2}{|c|}{ Cholesterol (mg/dl) } & \multicolumn{2}{|c|}{ LDL (mg/dl) } & \multicolumn{2}{|c|}{ HDL (mg/dl) } & \multicolumn{2}{|c|}{ Non-HDL (mg/dl) } & \multicolumn{2}{|c|}{ Triglycerides $^{*}(\mathrm{mg} / \mathrm{dl})$} \\
\hline & & Crude & Adjusted $^{\dagger Y}$ & Crude & Adjusted $^{\dagger}$ & Crude & Adjusted $^{\dagger}$ & Crude & Adjusted $^{\dagger}$ & Crude & Adjusted $^{\dagger}$ & Crude & Adjusted $^{\dagger}$ \\
\hline Breastfeeding & & $\mathrm{p}=0.50^{*}$ & $\mathrm{p}=0.95^{*}$ & $\mathrm{p}=0.07^{*}$ & $\mathrm{p}=0.96^{*}$ & $\mathrm{p}=0.13^{*}$ & $\mathrm{p}=0.83^{*}$ & $\mathrm{p}=0.09^{*}$ & $\mathrm{p}=0.24^{*}$ & $\mathrm{p}=0.19^{*}$ & $\mathrm{p}=0.70^{*}$ & $\mathrm{p}=0.59^{*}$ & $\mathrm{p}=0.42^{*}$ \\
\hline Ever & 1012 & $\operatorname{Ref}(0)$ & $\operatorname{Ref}(0)$ & $\operatorname{Ref}(0)$ & $\operatorname{Ref}(0)$ & $\operatorname{Ref}(0)$ & $\operatorname{Ref}(0)$ & $\operatorname{Ref}(0)$ & $\operatorname{Ref}(0)$ & $\operatorname{Ref}(0)$ & $\operatorname{Ref}(0)$ & $\operatorname{Ref}(1)$ & $\operatorname{Ref}(1)$ \\
\hline Never & 124 & $\begin{array}{l}-1.10 \\
(-5.6 ; 3.4)\end{array}$ & $\begin{array}{l}-0.40 \\
(-5.3 ; 4.5)\end{array}$ & $\begin{array}{l}7.17 \\
(0.2 ; 14.2)\end{array}$ & $\begin{array}{l}2.27 \\
(-6.8 \\
11.3)\end{array}$ & $\begin{array}{l}4.66 \\
(-1.0 ; \\
10.3)\end{array}$ & $\begin{array}{l}0.83 \\
(-6.6 ; 8.3)\end{array}$ & $\begin{array}{l}2.41 \\
(-0.1 ; 4.9)\end{array}$ & \begin{tabular}{|l|}
2.08 \\
$(-1.0 ; 5.2)$
\end{tabular} & $\begin{array}{l}4.76 \\
(-1.7 ; \\
11.2)\end{array}$ & $\begin{array}{l}0.19 \\
(-8.1 ; 8.5)\end{array}$ & $\begin{array}{l}1.04 \\
(0.9 ; 1.1)\end{array}$ & $\begin{array}{l}0.98 \\
(0.9 ; 1.1)\end{array}$ \\
\hline $1-<3$ & 88 & $\begin{array}{l}0.64 \\
(-4.6 ; 5.9)\end{array}$ & $\begin{array}{l}0.50 \\
(-5.8 ; 6.8)\end{array}$ & \begin{tabular}{|l|}
-2.08 \\
$(-10.1$ \\
$6.0)$ \\
\end{tabular} & $\begin{array}{l}2.58 \\
(-9.1 ; \\
14.2) \\
\end{array}$ & \begin{tabular}{|l|}
-3.66 \\
$(-10.1 ;$ \\
$2.8)$ \\
\end{tabular} & $\begin{array}{l}-1.60 \\
(-11.1 ; \\
7.9)\end{array}$ & $\begin{array}{l}2.56 \\
(-0.3 ; 5.5)\end{array}$ & $\begin{array}{l}2.85 \\
(-1.1 ; 6.8)\end{array}$ & \begin{tabular}{|l|}
-4.64 \\
$(-12.1 ;$ \\
$2.8)$ \\
\end{tabular} & $\begin{array}{l}-0.26 \\
(-11.0 ; \\
10.4) \\
\end{array}$ & $\begin{array}{l}0.99 \\
(0.9 ; 1.1)\end{array}$ & $\begin{array}{l}1.10 \\
(0.9 ; 1.3)\end{array}$ \\
\hline $3-<6$ & 142 & $\begin{array}{l}3.15 \\
(-1.1 ; 7.4)\end{array}$ & $\begin{array}{l}-0.60 \\
(-5.2 ; 4.0)\end{array}$ & $\begin{array}{l}3.35 \\
(-3.3 ; \\
10.0) \\
\end{array}$ & $\begin{array}{l}5.02 \\
(-3.4 ; \\
13.4) \\
\end{array}$ & $\begin{array}{l}3.01 \\
(-2.3 ; 8.3)\end{array}$ & $\begin{array}{l}3.98 \\
(-2.9 ; \\
10.9) \\
\end{array}$ & $\begin{array}{l}-0.32 \\
(-2.7 ; 2.1)\end{array}$ & $\begin{array}{l}0.27 \\
(-2.6 ; 3.1)\end{array}$ & $\begin{array}{l}3.67 \\
(-2.4 ; 9.8)\end{array}$ & $\begin{array}{l}4.75 \\
(-3.0 ; \\
12.5) \\
\end{array}$ & $\begin{array}{l}1.04 \\
(0.9 ; 1.1)\end{array}$ & $\begin{array}{l}1.07 \\
(0.9 ; 1.2)\end{array}$ \\
\hline$\geq 12$ & 519 & $\operatorname{Ref}(0)$ & $\operatorname{Ref}(0)$ & $\operatorname{Ref}(0)$ & $\operatorname{Ref}(0)$ & $\operatorname{Ref}(0)$ & $\operatorname{Ref}(0)$ & $\operatorname{Ref}(0)$ & $\operatorname{Ref}(0)$ & $\operatorname{Ref}(0)$ & $\operatorname{Ref}(0)$ & $\operatorname{Ref}(1)$ & $\operatorname{Ref}(1)$ \\
\hline Parity $=1$ & & \multicolumn{2}{|l|}{$\mathrm{P}=0.91^{*}$} & \multicolumn{2}{|l|}{$\mathrm{P}=0.61^{\ddagger}$} & \multicolumn{2}{|l|}{$\mathrm{P}=0.85^{\ddagger}$} & \multicolumn{2}{|l|}{$\mathrm{P}=0.52^{\ddagger}$} & \multicolumn{2}{|l|}{$\mathrm{P}=0.82^{\ddagger}$} & \multicolumn{2}{|l|}{$\mathrm{P}=0.31^{*}$} \\
\hline $\begin{array}{l}\text { Total sum of } \\
\text { breastfeeding }\end{array}$ & & $\mathrm{P}=0.60^{*}$ & $\mathrm{p}=0.73^{*}$ & $\mathrm{P}=0.34^{*}$ & $\mathrm{P}=0.46^{* *}$ & $\mathrm{P}=0.47^{* *}$ & $\mathrm{P}=0.87^{*}$ & $\mathrm{P}=0.11^{*}$ & $\mathrm{P}=0.28^{*} *$ & $\mathrm{P}=0.35^{* *}$ & $\mathrm{P}=0.68^{* *}$ & $\mathrm{P}=0.18^{* *}$ & $\mathrm{P}=0.25^{*}$ \\
\hline Never & 97 & $\begin{array}{l}0.06 \\
(-6.2 ; 6.3)\end{array}$ & $\begin{array}{l}1.03 \\
(-4.8 ; 6.9)\end{array}$ & $\begin{array}{l}6.62 \\
(-2.1 ; \\
15.3)\end{array}$ & $\begin{array}{l}5.64 \\
(-6.1 ; \\
17.4)\end{array}$ & $\begin{array}{l}4.87 \\
(-2.3 ; \\
12.0)\end{array}$ & $\begin{array}{l}1.98 \\
(-7.6 ; \\
11.6)\end{array}$ & $\begin{array}{l}0.52 \\
(-2.6 ; 3.6)\end{array}$ & $\begin{array}{l}2.47 \\
(-1.5 ; 6.4)\end{array}$ & $\begin{array}{l}6.10 \\
(-2.1 ; \\
14.3)\end{array}$ & $\begin{array}{l}3.17 \\
(-7.7 ; \\
14.0)\end{array}$ & $\begin{array}{l}1.08 \\
(0.9 ; 1.2)\end{array}$ & $\begin{array}{l}1.03 \\
(0.9 ; 1.2)\end{array}$ \\
\hline $1-<3$ & 71 & $\begin{array}{l}2.05 \\
(-4.9 ; 9.0)\end{array}$ & $\begin{array}{l}2.56 \\
(-4.4 ; 9.5)\end{array}$ & $\begin{array}{l}-3.08 \\
(-12.7 \\
6.6)\end{array}$ & $\begin{array}{l}5.36 \\
(-8.6 ; \\
19.3)\end{array}$ & $\begin{array}{l}-2.94 \\
(-10.9 ; \\
5.0)\end{array}$ & $\begin{array}{l}-0.10 \\
(-11.5 ; \\
11.3)\end{array}$ & $\begin{array}{l}-0.85 \\
(-4.3 ; 2.6)\end{array}$ & $\begin{array}{l}2.08 \\
(-2.6 ; 6.8)\end{array}$ & $\begin{array}{l}-2.23 \\
(-11.3 ; \\
6.8)\end{array}$ & $\begin{array}{l}3.28 \\
(-9.6 ; \\
16.1)\end{array}$ & $\begin{array}{l}1.08 \\
(0.9 ; 1.2)\end{array}$ & $\begin{array}{l}1.20 \\
(0.9 ; 1.5)\end{array}$ \\
\hline $3-<6$ & 108 & $\begin{array}{l}4.70 \\
(-1.3 ; \\
10.7) \\
\end{array}$ & $\begin{array}{l}-0.44 \\
(-5.9 ; 5.0)\end{array}$ & $\begin{array}{l}-1.23 \\
(-9.6 ; 7.2)\end{array}$ & $\begin{array}{l}2.05 \\
(-8.9 ; \\
13.0) \\
\end{array}$ & $\begin{array}{l}0.56 \\
(-6.3 ; 7.4)\end{array}$ & $\begin{array}{l}1.16 \\
(-7.8 ; \\
10.1) \\
\end{array}$ & $\begin{array}{l}-3.16 \\
(-6.1 ; \\
-0.2)\end{array}$ & $\begin{array}{l}-0.44 \\
(-4.1 ; 3.2)\end{array}$ & $\begin{array}{l}1.93 \\
(-5.9 ; 9.8)\end{array}$ & $\begin{array}{l}2.48 \\
(-7.6 ; \\
12.6)\end{array}$ & $\begin{array}{l}1.08 \\
(0.9 ; 1.2)\end{array}$ & $\begin{array}{l}1.12 \\
(0.9 ; 1.3)\end{array}$ \\
\hline Never & 27 & \begin{tabular}{|l|}
-3.31 \\
$(-11.4 ;$ \\
$4.7)$
\end{tabular} & $\begin{array}{l}-2.64 \\
(-10.7 ; \\
5.4)\end{array}$ & $\begin{array}{l}-3.66 \\
(-17.7 ; \\
10.4)\end{array}$ & $\begin{array}{l}-15.83 \\
(-33.0 ; \\
1.4)\end{array}$ & $\begin{array}{l}-0.63 \\
(-11.8 \\
10.6)\end{array}$ & $\begin{array}{l}-11.34 \\
(-25.6 ; \\
3.0)\end{array}$ & $\begin{array}{l}-1.43 \\
(-6.4 ; 3.5)\end{array}$ & $\begin{array}{l}-0.96 \\
(-6.9 ; 5.0)\end{array}$ & $\begin{array}{l}-2.23 \\
(-15.2 \\
10.8)\end{array}$ & $\begin{array}{l}-14.87 \\
(-30.7 ; \\
1.0)\end{array}$ & $\begin{array}{l}0.97 \\
(0.8 ; 1.2)\end{array}$ & \begin{tabular}{|l|l}
0.90 \\
$(0.7 ; 1.1)$
\end{tabular} \\
\hline $1-<3$ & 17 & $\begin{array}{l}-2.95 \\
(-12.9 ; \\
7.0)\end{array}$ & $\begin{array}{l}-7.55 \\
(-21.2 \\
6.1) \\
\end{array}$ & $\begin{array}{l}-13.27 \\
(-30.7 \\
4.2)\end{array}$ & \begin{tabular}{|l}
-13.80 \\
$(-43.1$ \\
$15.5)$
\end{tabular} & $\begin{array}{l}-12.10 \\
(-26.0 \\
1.8)\end{array}$ & $\begin{array}{l}-14.17 \\
(-38.5 ; \\
10.2)\end{array}$ & $\begin{array}{l}4.03 \\
(-2.1 ; \\
10.2)\end{array}$ & $\begin{array}{l}4.04 \\
(-6.0 ; \\
14.1)\end{array}$ & $\begin{array}{l}-17.3 \\
(-33.5 ; \\
-1.2)\end{array}$ & $\begin{array}{l}-17.84 \\
(-44.8 ; \\
9.1)\end{array}$ & $\begin{array}{l}0.80 \\
(0.6 ; 1.0)\end{array}$ & $\begin{array}{l}0.94 \\
(0.6 ; 1.4)\end{array}$ \\
\hline $3-<6$ & 34 & $\begin{array}{l}-0.10 \\
(-7.3 ; 7.1)\end{array}$ & \begin{tabular}{|l|}
2.29 \\
$(-5.4$ \\
$10.0)$
\end{tabular} & $\begin{array}{l}6.56 \\
(-6.1 ; \\
19.2)\end{array}$ & $\begin{array}{l}10.17 \\
(-6.0 \\
26.3)\end{array}$ & $\begin{array}{l}6.72 \\
(-3.3 ; \\
16.8)\end{array}$ & $\begin{array}{l}7.27 \\
(-6.1 ; \\
20.7)\end{array}$ & $\begin{array}{l}-0.61 \\
(-5.1 ; 3.8)\end{array}$ & $\begin{array}{l}0.39 \\
(-5.2 ; 5.9)\end{array}$ & $\begin{array}{l}7.17 \\
(-4.5 ; \\
18.9)\end{array}$ & $\begin{array}{l}9.78 \\
(-5.1 ; \\
24.6)\end{array}$ & $\begin{array}{l}0.99 \\
(0.8 ; 1.2)\end{array}$ & $\begin{array}{l}1.08 \\
(0.9 ; 1.3)\end{array}$ \\
\hline $6-<12$ & 111 & $\begin{array}{l}-2.20 \\
(-6.6 ; 2.2)\end{array}$ & $\begin{array}{l}-5.47 \\
(-10.4 ; \\
-0.5)\end{array}$ & $\begin{array}{l}-1.40 \\
(-9.1 ; 6.3)\end{array}$ & $\begin{array}{l}-1.33 \\
(-11.9 ; \\
9.2)\end{array}$ & $\begin{array}{l}0.24 \\
(-5.9 ; 6.4)\end{array}$ & $\begin{array}{l}3.48 \\
(-5.3 ; \\
12.2)\end{array}$ & $\begin{array}{l}-2.51 \\
(-5.2 ; 0.2)\end{array}$ & $\begin{array}{l}-1.93 \\
(-5.6 ; 1.7)\end{array}$ & $\begin{array}{l}1.10 \\
(-6.0 ; 8.3)\end{array}$ & $\begin{array}{l}0.60 \\
(-9.1 ; \\
10.3)\end{array}$ & $\begin{array}{l}0.99 \\
(0.9 ; 1.1)\end{array}$ & $\begin{array}{l}0.91 \\
(0.8 ; 1.1)\end{array}$ \\
\hline$\geq 12$ & 348 & $\operatorname{Ref}(0)$ & $\operatorname{Ref}(0)$ & $\operatorname{Ref}(0)$ & $\operatorname{Ref}(0)$ & $\operatorname{Ref}(0)$ & $\operatorname{Ref}(0)$ & $\operatorname{Ref}(0)$ & $\operatorname{Ref}(0)$ & $\operatorname{Ref}(0)$ & $\operatorname{Ref}(0)$ & $\operatorname{Ref}(1)$ & $\operatorname{Ref}(1)$ \\
\hline
\end{tabular}

Table 3. Glycaemia and lipid profile according to total breastfeeding $(\mathrm{n}=1136)$. Pelotas, 2012. ${ }^{*}$ Logtransformed data (multiplicative effect). ${ }^{\dagger}$ Adjusted for genomic ancestry, family income and maternal schooling at birth; asset index at childhood; and family income, schooling, asset index, energy intake, physical activity, alcohol consumption, current smoking and BMI at 2004-5. ${ }^{*}$ Glycaemia was also adjusted for use of hypoglycemic drugs. *Test for heterogeneity. **Test for linear trend. ${ }^{*} \mathrm{p}$-value for interaction.

lipids ${ }^{12,16,19,22}$, whereas others have not observed such associations ${ }^{12,18,23}$. Concerning the carotid intima-media thickness, one study showed a positive association ${ }^{31}$ and two others did not observe such association ${ }^{32,33}$, while for pulse wave velocity one study did not found an association ${ }^{32}$. As mentioned before, most of the published studies have been carried out in high income countries, where breastfeeding is positively associated with socioeconomic status $^{5}$, and adjusted for few socioeconomic variables. In this context, the estimates could be biased by residual confounding by socioeconomic status. In our study, breastfeeding was inversely associated with socioeconomic status, and we adjusted the estimates for several socioeconomic factors to minimize the possibility of residual confounding. Therefore, our results were probably not due to residual confounding. However, considering the 


\begin{tabular}{|c|c|c|c|c|c|}
\hline & \multirow[b]{2}{*}{$\mathbf{N}$} & \multicolumn{4}{|c|}{ Adjusted regression coefficient ( $95 \%$ confidence interval) $\dagger$} \\
\hline & & \begin{tabular}{|l|}
$\begin{array}{l}\text { Systolic blood } \\
\text { pressure }^{\Psi}(\mathbf{m m H g})\end{array}$
\end{tabular} & \begin{tabular}{|l|} 
Diastolic blood \\
pressure $(\mathrm{mmHg})$
\end{tabular} & $\begin{array}{l}\text { Intima-media } \\
\text { thickness }(\mu \mathrm{m})\end{array}$ & $\begin{array}{l}\text { Pulse wave } \\
\text { velocity }(\mathrm{m} / \mathrm{s})\end{array}$ \\
\hline \multicolumn{6}{|c|}{ Last child breastfeeding } \\
\hline Time since last birth & & $\mathrm{P}=0.21^{\ddagger}$ & $\mathrm{P}=0.07^{\ddagger}$ & $\mathrm{P}=0.25^{\ddagger}$ & $\mathrm{P}=0.86^{\ddagger}$ \\
\hline$<5$ years & & $\mathrm{p}=0.34^{*}$ & $\mathrm{p}=0.09^{*}$ & $\mathrm{P}=0.10^{*}$ & $\mathrm{P}=0.25^{*}$ \\
\hline Never & 68 & $1.82(-1.9 ; 5.5)$ & $2.26(-0.4 ; 4.9)$ & $-3.90(-8.6 ; 0.8)$ & $0.32(-0.2 ; 0.9)$ \\
\hline Ever & 489 & $\operatorname{Ref}(0)$ & $\operatorname{Ref}(0)$ & $\operatorname{Ref}(0)$ & $\operatorname{Ref}(0)$ \\
\hline$\geq 5$ years & & $\mathrm{p}=0.59^{*}$ & $\mathrm{p}=0.55^{*}$ & $\mathrm{P}=0.86^{*}$ & $\mathrm{P}=0.79^{*}$ \\
\hline Never & 84 & $-1.04(-4.8 ; 2.7)$ & $-0.86(-3.7 ; 1.9)$ & $0.53(-5.3 ; 6.4)$ & $0.07(-0.4 ; 0.6)$ \\
\hline Ever & 492 & $\operatorname{Ref}(0)$ & $\operatorname{Ref}(0)$ & $\operatorname{Ref}(0)$ & $\operatorname{Ref}(0)$ \\
\hline \multicolumn{6}{|c|}{ Total last child breastfeeding (months) } \\
\hline Time since last birth & & $\mathrm{P}=0.13^{\ddagger}$ & $\mathrm{P}=0.15^{\ddagger}$ & $\mathrm{P}=0.24^{\ddagger}$ & $\mathrm{P}=0.95^{\ddagger}$ \\
\hline$<5$ years & & $\mathrm{p}=0.17^{*}$ & $\mathrm{p}=0.21^{*}$ & $\mathrm{P}=0.16^{*}$ & $\mathrm{P}=0.47 * *$ \\
\hline Never & 68 & $1.02(-2.8 ; 4.8)$ & $1.88(-0.8 ; 4.6)$ & $-3.51(-8.3 ; 1.3)$ & $0.28(-0.3 ; 0.9)$ \\
\hline $1-<3$ & 66 & $-1.44(-5.5 ; 2.6)$ & $-1.68(-4.6 ; 1.2)$ & $4.16(-1.2 ; 9.5)$ & $-0.08(-0.8 ; 0.7)$ \\
\hline $3-<6$ & 95 & $-3.36(-6.7 ;-0.5)$ & $-1.06(-3.4 ; 1.3)$ & $-0.17(-4.4 ; 4.0)$ & $-0.15(-0.7 ; 0.4)$ \\
\hline$\geq 6$ & 328 & $\operatorname{Ref}(0)$ & $\operatorname{Ref}(0)$ & $\operatorname{Ref}(0)$ & $\operatorname{Ref}(0)$ \\
\hline$\geq 5$ years & & $\mathrm{P}=0.28^{* *}$ & $\mathrm{p}=0.28^{* *}$ & $\mathrm{P}=0.57^{*}$ & $\mathrm{P}=0.64^{* *}$ \\
\hline Never & 84 & $-1.87(-5.8 ; 2.1)$ & $-1.37(-4.3 ; 1.6)$ & $0.65(-5.4 ; 6.7)$ & $0.07(-0.5 ; 0.6)$ \\
\hline $1-<3$ & 80 & $-1.48(-5.8 ; 2.9)$ & $-1.10(-4.3 ; 2.1)$ & $3.68(-2.8 ; 10.2)$ & $0.22(-0.4 ; 0.8)$ \\
\hline $3-<6$ & 106 & $-2.41(-5.9 ; 1.1)$ & $-1.41(-4.0 ; 1.2)$ & $-1.57(-6.8 ; 3.6)$ & $-0.09(-0.5 ; 0.3)$ \\
\hline$\geq 6$ & 306 & $\operatorname{Ref}(0)$ & $\operatorname{Ref}(0)$ & $\operatorname{Ref}(0)$ & $\operatorname{Ref}(0)$ \\
\hline
\end{tabular}

Table 4. Blood pressure, carotid intima-media thickness and pulse wave velocity according to last child breastfeeding and stratified to time since last birth $(n=1133)$. Pelotas, $2012 .{ }^{\dagger}$ Adjusted for genomic ancestry, family income and maternal schooling at birth; asset index at childhood; family income, schooling, asset index, energy intake, physical activity, alcohol consumption, current smoking and BMI at 2004-5; and parity. ${ }^{\ddagger}$ Systolic and diastolic blood pressure were also adjusted for use of hypertensive drugs. ${ }^{\ddagger}$ p-value for interaction. ${ }^{*}$ Test for heterogeneity. ${ }^{* *}$ Test for linear trend.

natural history of the risk factors evaluated, our studied population is young, and these morbidities may not yet be clinically detectable, which may explain in part our negative findings.

Studies have evaluated weather time since last childbirth modifies the association of breastfeeding with cardiovascular risk factors. Gunderson et al., using data from the Coronary Artery Risk Development in Young Adults, assessed the effect of breastfeeding on subclinical atherosclerosis and observed no interaction $(\mathrm{p} \geq 0.1)^{31}$. Stuebe et al. also reported a non-significant interaction with time since last birth when evaluating parous women from the Nurses' Health Study (NHS) and Nurses' Health Study II (NHS II) (NHS: $p=0.32$; NHS II: $p=0.54$ ), despite having observed association between breastfeeding and type II diabetes in women who gave birth in the past 15 years in both NHS and NHSII and no association in those with time higher than 15 in the NHS II and a reduced association in the $\mathrm{NHS}^{29}$. When assessing the association of breastfeeding and maternal cardiovascular disease in postmenopausal women from the Women's Health Initiative, Schwarz et al. verified a significative interaction with age $(p=0.02)$, but no with age at last lactation $(p=0.58)^{19}$. In addition, other studies showed that the association of breastfeeding with metabolic risk factors was weaker in women with longer time since last birth ${ }^{9,30}$ and declined as age increased ${ }^{15,16,34,35}$; but in the latter the effect modification may be due to time since last birth, and not properly due to age.

One limitation of observational studies is that residual confounding may bias the estimates. In contrast, exchangeability between the comparison groups is expected in experimental studies. Oken et al. ${ }^{17}$ evaluated the effect of breastfeeding on maternal blood pressure using data from the Promotion of Breastfeeding Intervention Trial (PROBIT), in which hospitals and polyclinics from Belarus were randomized to implement the Baby-Friendly Hospital Initiative. On intention-to-treat analysis, they observed lower levels of systolic (mean difference: $-0.81,95 \% \mathrm{CI}:-3.33 ; 1.71$ ) and diastolic blood pressure (mean difference: $-1.09,95 \% \mathrm{CI}:-2.43 ; 0.25$ ) and odds of hypertension (OR: $0.85,95 \% \mathrm{CI}: 0.64 ; 1.12$ ) in the intervention arm compared to the control, but these associations were not statistical significant. However, one limitation of this study is that the compliance was low, that is women from the intervention group did not breastfeed their child while some from the control group breastfed, which reduced the power to detect differences between the groups. Therefore, the non-statistically significant association may not be due to the nonexistence of an association.

Our study has several strengths. It was based on information from a large birth cohort with a high follow-up rate. We were able to evaluate the association of breastfeeding with several metabolic risk factors and all measures were obtained by trained interviewers. Also, the information on confounding factors was collected prospectively, reducing the chance of residual confounding. Although the attrition rate was slightly higher among those in the extreme socioeconomic categories, breastfeeding per livebirth was independent of socioeconomic status, so this small difference is unlikely of having introduced a selection bias. However, some limitations must be considered. We were not able to adjust for some possible confounding factors, such as pre-gestational body mass index and 


\begin{tabular}{|c|c|c|c|c|c|c|c|}
\hline & \multirow[b]{2}{*}{$\mathbf{N}$} & \multicolumn{6}{|c|}{ Adjusted regression coefficient $(95 \% \text { confidence interval })^{\dagger}$} \\
\hline & & Glycaemia $^{\mathrm{x}}(\mathrm{mg} / \mathrm{dl})$ & Cholesterol (mg/dl) & LDL (mg/dl) & HDL (mg/dl) & Non-HDL (mg/dl) & Triglycerides $^{*}(\mathrm{mg} / \mathrm{dl})$ \\
\hline \multicolumn{8}{|c|}{ Last child breastfeeding } \\
\hline Time since last birth & & $\mathrm{P}=0.75^{\ddagger}$ & $\mathrm{P}=0.67^{\ddagger}$ & $\mathrm{P}=0.44^{\ddagger}$ & $\mathrm{P}=0.77^{\ddagger}$ & $\mathrm{P}=0.57^{\ddagger}$ & $\mathrm{P}=0.86^{\ddagger}$ \\
\hline$<5$ years & & $\mathrm{p}=0.66^{*}$ & $\mathrm{P}=0.88^{*}$ & $\mathrm{P}=0.82^{*}$ & $\mathrm{P}=0.65^{*}$ & $\mathrm{P}=0.99^{*}$ & $\mathrm{P}=0.73^{*}$ \\
\hline Never & 68 & $-1.37(-7.4 ; 4.7)$ & $0.88(-10.7 ; 12.5)$ & $1.14(-8.6 ; 10.8)$ & $0.89(-2.9 ; 4.7)$ & $-0.01(-10.9 ; 10.9)$ & $0.97(0.8 ; 1.2)$ \\
\hline Ever & 489 & $\operatorname{Ref}(0)$ & $\operatorname{Ref}(0)$ & $\operatorname{Ref}(0)$ & $\operatorname{Ref}(0)$ & $\operatorname{Ref}(0)$ & $\operatorname{Ref}(1)$ \\
\hline$\geq 5$ years & & $\mathrm{p}=0.66^{*}$ & $\mathrm{P}=0.87^{*}$ & $\mathrm{P}=0.53^{*}$ & $\mathrm{P}=0.19^{*}$ & $\mathrm{P}=0.49^{*}$ & $\mathrm{P}=0.40^{*}$ \\
\hline Never & 84 & $-1.20(-6.5 ; 4.1)$ & $-0.91(-12.2 ; 10.4)$ & $-2.87(-11.9 ; 6.2)$ & $2.64(-1.3 ; 6.6)$ & $-3.55(-13.6 ; 6.5)$ & $0.94(0.8 ; 1.1)$ \\
\hline Ever & 492 & $\operatorname{Ref}(0)$ & $\operatorname{Ref}(0)$ & $\operatorname{Ref}(0)$ & $\operatorname{Ref}(0)$ & $\operatorname{Ref}(0)$ & $\operatorname{Ref}(1)$ \\
\hline \multicolumn{8}{|c|}{ Total last child breastfeeding (months) } \\
\hline Time since last birth & & $\mathrm{P}=0.67^{\ddagger}$ & $\mathrm{P}=0.51^{*}$ & $\mathrm{P}=0.83^{\ddagger}$ & $\mathrm{P}=0.19^{\ddagger}$ & $\mathrm{P}=0.83^{\ddagger}$ & $\mathrm{P}=0.74^{\ddagger}$ \\
\hline$<5$ years & & $\mathrm{p}=0.34^{* *}$ & $\mathrm{P}=0.32 * *$ & $\mathrm{P}=0.52^{* *}$ & $\mathrm{P}=0.15^{* *}$ & $\mathrm{P}=0.58^{* *}$ & $\mathrm{P}=0.73^{*}$ \\
\hline Never & 68 & $-2.11(-8.4 ; 4.1)$ & $2.77(-9.1 ; 14.7)$ & $1.98(-8.0 ; 12.0)$ & $1.84(-2.1 ; 5.7)$ & $0.94(-10.3 ; 12.2)$ & $0.98(0.8 ; 1.2)$ \\
\hline $1-<3$ & 66 & $-2.89(-9.7 ; 3.9)$ & $8.45(-4.5 ; 21.4)$ & $3.66(-7.2 ; 14.5)$ & $2.51(-1.7 ; 6.7)$ & $5.93(-6.3 ; 18.1)$ & $1.11(0.9 ; 1.3)$ \\
\hline $3-<6$ & 95 & $-2.17(-7.7 ; 3.4)$ & $4.76(-5.8 ; 15.3)$ & $2.15(-6.7 ; 11.0)$ & $3.46(0.1 ; 6.9)$ & $1.30(-8.7 ; 11.3)$ & $1.00(0.9 ; 1.2)$ \\
\hline$\geq 6$ & 328 & $\operatorname{Ref}(0)$ & $\operatorname{Ref}(0)$ & $\operatorname{Ref}(0)$ & $\operatorname{Ref}(0)$ & $\operatorname{Ref}(0)$ & $\operatorname{Ref}(1)$ \\
\hline$\geq 5$ years & & $\mathrm{p}=0.70^{* *}$ & $\mathrm{P}=0.92^{* *}$ & $\mathrm{P}=0.70^{*}$ & $\mathrm{P}=0.33^{*}$ & $\mathrm{P}=0.81^{*}$ & $\mathrm{P}=0.18^{*}$ \\
\hline Never & 84 & $-1.36(-6.9 ; 4.2)$ & $-0.38(-12.2 ; 11.4)$ & $-1.87(-11.4 ; 7.6)$ & $2.12(-2.0 ; 6.2)$ & $-2.50(-13.0 ; 8.1)$ & $0.95(0.8 ; 1.1)$ \\
\hline $1-<3$ & 80 & $0.23(-5.9 ; 6.3)$ & $2.44(-10.5 ; 15.3)$ & $-0.38(-10.7 ; 10.0)$ & $0.27(-4.2 ; 4.7)$ & $2.18(-9.4 ; 13.7)$ & $1.17(0.9 ; 1.4)$ \\
\hline $3-<6$ & 106 & $-0.72(-5.6 ; 4.1)$ & $0.83(-9.4 ; 11.1)$ & $4.11(-4.1 ; 12.4)$ & $-2.21(-5.8 ; 1.3)$ & $3.04(-6.1 ; 12.2)$ & $0.97(0.9 ; 1.1)$ \\
\hline$\geq 6$ & 306 & $\operatorname{Ref}(0)$ & $\operatorname{Ref}(0)$ & $\operatorname{Ref}(0)$ & $\operatorname{Ref}(0)$ & $\operatorname{Ref}(0)$ & $\operatorname{Ref}(1)$ \\
\hline
\end{tabular}

Table 5. Glycaemia and lipid profile according to last child breastfeeding and stratified to time since last birth $(\mathrm{n}=1133)$. Pelotas, 2012. ${ }^{*}$ Log-transformed data (multiplicative effect). ${ }^{\dagger}$ Adjusted for genomic ancestry, family income and maternal schooling at birth; asset index at childhood; family income, schooling, asset index, energy intake, physical activity, alcohol consumption, current smoking and BMI at 2004-5; and parity. ${ }^{\ddagger}$ Glycaemia was also adjusted for use of hypoglycemic drugs. ${ }^{*}$ p-value for interaction. ${ }^{*}$ Test for heterogeneity. ${ }^{* *}$ Test for linear trend.

weight gain during pregnancy, because we did not collect information on these variables. For the same reason, we could not adjust for smoking or household exposure to tobacco in pregnancy. When controlling the analysis for tobacco smoking in the 2004-5 as a proxy for those variables, the magnitude of the differences barely decreased. As people hardly quit smoking due to addiction, and it is not expected that non-smokers women start the habit during pregnancy, we believe it is not likely that our results are due to confounding for tobacco in pregnancy. We do not have data on patterns and daily frequency of lactation, and also could not distinguish between direct breastfeeding and pumped breast milk, so our findings should be interpreted with caution. Additionally, glycaemia was evaluated using non-fasting blood glucose measure. Even having adjusted for time since last meal, it may have introduced a non-differential misclassification, but the magnitude of the association was small. Therefore, it is unlikely that this negative result was due to the misclassification.

In conclusion, our findings suggest that there is no association between lactation and maternal cardiometabolic risk factors.

\section{Materials and Methods}

Participants. This study is based on data from the 1982 Pelotas Birth Cohort Study. In 1982, all the maternity hospitals located in Pelotas, a southern Brazilian city, were visited daily and all births identified $(\mathrm{n}=7392)$. Based on data from birth registration and a city census, we estimated that our hospital sample accounts for $99.2 \%$ of all births in the city. Those live births whose family lived in the urban area of the city were examined and their mothers interviewed soon after delivery $(n=5914)$. These subjects have been prospectively followed at different ages. Further details on the study methodology have been published elsewhere ${ }^{36,37}$. From June 2012 to February 2013, we tried to follow the whole cohort. Multiple strategies were used to locate the study participants, who were invited to attend the research clinic to be interviewed, examined, and provided a blood sample ${ }^{38}$. Of the 5914 members of the 1982 Cohort, 3701 agreed to participate, which added to the 325 deaths identified among the cohort participants, represented a follow-up rate of $68.1 \%$. In the present study we included only women evaluated in 2012-13, who had a previous delivery, and were not pregnant when interviewed $(\mathrm{n}=1147)$.

Exposure. In 2012-13 visit, the subjects provided the following information on each offspring: birthweight, type of delivery and duration of breastfeeding. Cumulative lifetime duration of breastfeeding (in months) was obtained by summing the duration of lactation of all offspring. We also assess the duration of breastfeeding of the last child.

Outcomes. In the present study, we evaluated the following outcomes that were evaluated in the 2012-13 visit: 
- Blood pressure was measured twice, at the beginning and at the end of the anthropometrical assessment, on the left arm, using an automatic digital sphygmomanometer, model Omron HEM 705CPINT, with a specific cuff for obese individuals. The mean of the measurements was used in the analysis.

- Carotid intima-media thickness of the posterior wall of right and left carotid arteries was evaluated in longitudinal planes using a Toshiba Xario ultrasound ${ }^{39}$. A $10 \mathrm{~mm}$-long section of the common carotid artery was imaged proximal to carotid bulb using the Carotid Analyzer for Research, Medical Imaging Application-LLC, that evaluated the arithmetic mean of 90 frames. The mean of the measurements was used in the analysis.

- Pulse wave velocity was assessed using a portable ultrasound, Sphygmocor ${ }^{\circledR}$ (AtCor Medical, Version 9.0, Sydney, Australia) in supine position, after resting for 5 minutes. Pulse wave velocity was estimated by dividing the distance between carotid and femoral sites by the transit time between the carotid and femoral pulse wave.

- Random blood glucose was measured using an automatic enzymatic colorimetric method, BS-380, Mindray (Shenzhen Mindray Bio-Medical Electronics Co., Ltd, China), and the assay sensitivity was $1.31 \mathrm{mg} / \mathrm{dl}$. Because glucose levels vary according to fasting time, estimates were adjusted for time since the last meal ${ }^{40}$.

- HDL, LDL, total cholesterol and triglycerides were measured using an enzymatic assay (Shenzhen Mindray Bio-Medical Electronics Co., Ltd, China), and the assays sensitivity was $2.9996 \mathrm{mg} / \mathrm{dl}, 0.2540 \mathrm{mg} / \mathrm{dl}, 1.472 \mathrm{mg} /$ $\mathrm{dl}$ and $2.845 \mathrm{mg} / \mathrm{dl}$, respectively. Non-HDL was obtained by subtracting HDL from total cholesterol.

Confounders. The following variables were considered as possible confounders. Family income in minimum wages and maternal schooling (in complete years of schooling) at birth were provided by the mother in the perinatal study. A household asset index in childhood was estimated using principal component analysis and based on household characteristics, such as type of building, piped water in the household, type of lavatory, presence of a gas stove at home, wood stove at home and number of bedrooms. Other variables collected during the 2004-5 follow-up visits were used in the analysis, such as family income (in Brazilian reais), schooling, asset index according to criteria of the Brazilian Association of Research Companies, European genomic ancestry (based on approximately 370,000 SNPs mutually available for the Pelotas cohort and selected samples of the HapMap and Human Genome Diversity - ADMIXTURE was used to estimate the genomic ancestry of each subject ${ }^{41}$, daily energy intake based on a food frequency questionnaire with recall period of 12 months, leisure time physical activity assessed through the International Physical Activity Questionnaire (minutes/week) ${ }^{42}$, alcohol consumption in the last week, self-reported tobacco smoking and body mass index. When evaluating systolic and diastolic blood pressure and glycaemia, we also adjusted for treatment with antihypertensive and hypoglycemic drugs, respectively.

Statistical analyses. Analysis of variance (ANOVA) and chi-square test were used to assess differences between means and proportions, respectively. Because all the outcomes were continuous, linear regression was used to evaluate the association of breastfeeding duration with maternal metabolic cardiovascular risk factors. Triglycerides were log-transformed because its distribution was asymmetric. Statistical comparisons between groups were based on tests of heterogeneity and linear trend, and the one with the lower p-value was presented. In the multivariable analysis, estimates were adjusted for biological, socioeconomic and behavioral variables. The analysis was also stratified for parity. When evaluating the last child breastfeeding, we adjusted for parity and stratified the analysis by time since last birth. We used Stata 13.0 for the analyses.

Ethics statement. All participants signed a written informed consent and the Research Ethics Committee of the Faculty of Medicine, Federal University of Pelotas, approved the study protocol (protocol number: Of.16/12). All methods were performed in accordance with relevant guidelines and regulations.

\section{Data Availability}

The dataset is available from the corresponding author on reasonable request.

\section{References}

1. Sankar, M. J. et al. Optimal breastfeeding practices and infant and child mortality: a systematic review and meta-analysis. Acta Paediatr 104, 3-13 (2015).

2. Horta, B. L., de Mola, C. L. \& Victora, C. G. Long-term consequences of breastfeeding on cholesterol, obesity, systolic blood pressure and type 2 diabetes: a systematic review and meta-analysis. Acta Paediatr 104, 30-37 (2015).

3. Horta, B. L., de Mola, C. L. \& Victora, C. G. Breastfeeding and intelligence: a systematic review and meta-analysis. Acta Paediatr 104, 14-19 (2015).

4. Chowdhury, R. et al. Breastfeeding and maternal health outcomes: a systematic review and meta-analysis. Acta Paediatr 104, 96-113 (2015).

5. Victora, C. G. et al. Breastfeeding in the 21st century: epidemiology, mechanisms, and lifelong effect. Lancet 387, 475-490 (2016).

6. Stuebe, A. M. et al. Duration of lactation and incidence of myocardial infarction in middle to late adulthood. Am J Obstet Gynecol 200, 138.e1-8 (2009).

7. Peters, S. A. E. et al. Breastfeeding and the Risk of Maternal Cardiovascular Disease: A Prospective Study of 300000 Chinese Women. J Am Heart Assoc 6, e006081 (2017).

8. Aune, D., Norat, T., Romundstad, P. \& Vatten, L. J. Breastfeeding and the maternal risk of type 2 diabetes: A systematic review and dose response meta-analysis of cohort studies. Nutr Metab Cardiovasc Dis 24, 107-115 (2014).

9. Jager, S. et al. Breast-feeding and maternal risk of type 2 diabetes: a prospective study and meta-analysis. Diabetologia 57, 1355-1365 (2014).

10. Gunderson, E. P. et al. Lactation Duration and Progression to Diabetes in Women Across the Childbearing Years: The 30-Year CARDIA Study. JAMA Intern Med. 178, 328-337 (2018).

11. Groer, M. W., Jevitt, C. M., Sahebzamani, F., Beckstead, J. W. \& Keefe, D. L. Breastfeeding Status and Maternal Cardiovascular Variables Across the Postpartum. J Womens Health (Larchmt) 22, 453-459 (2013).

12. Gunderson, E. P. et al. Lactation and changes in maternal metabolic risk factors. Obstet Gynecol 109, 729-738 (2007). 
13. Ebina, S. \& Kashiwakura, I. Influence of breastfeeding on maternal blood pressure at one month postpartum. Int J Womens Health 4, 333-339 (2012).

14. Lee, S. Y., Kim, M. T., Jee, S. H. \& Yang, H. P. Does long-term lactation protect premenopausal women against hypertension risk? a Korean women's cohort study. Prev Med 41, 433-438 (2005).

15. Lupton, S. J., Chiu, C. L., Lujic, S., Hennessy, A. \& Lind, J. M. Association between parity and breastfeeding with maternal high blood pressure. Am J Obstet Gynecol 208, 454.e1-454.e7 (2013).

16. Natland, S. T., Nilsen, T. I., Midthjell, K., Andersen, L. F. \& Forsmo, S. Lactation and cardiovascular risk factors in mothers in a population-based study: the HUNT-study. Int Breastfeed J 7, 8 (2012).

17. Oken, E. et al. Effects of an intervention to promote breastfeeding on maternal adiposity and blood pressure at $11.5 \mathrm{y}$ postpartum: results from the Promotion of Breastfeeding Intervention Trial, a cluster-randomized controlled trial. Am J Clin Nutr 98, 1048-1056 (2013).

18. Ram, K. T. et al. Duration of lactation is associated with lower prevalence of the metabolic syndrome in midlife - SWAN, the study of women's health across the nation. Am J Obstet Gynecol 198, 268.e1-268.e6 (2008).

19. Schwarz, E. B. et al. Duration of Lactation and Risk Factors for Maternal Cardiovascular Disease. Obstet Gynecol 113, 974-982 (2009).

20. Stuebe, A. M. et al. Duration of Lactation and Incidence of Maternal Hypertension: A Longitudinal Cohort Study. Am J Epidemiol 174, 1147-1158 (2011).

21. Zhang, B. Z., Zhang, H. Y., Liu, H. H., Li, H. J. \& Wang, J. S. Breastfeeding and Maternal Hypertension and Diabetes: A PopulationBased Cross-Sectional Study. Breastfeed Med 10, 163-167 (2015).

22. Wiklund, P. et al. Prolonged breast-feeding protects mothers from later-life obesity and related cardio-metabolic disorders. Public Health Nutr 15, 67-74 (2012).

23. Stuebe, A. M. et al. Duration of Lactation and Maternal Metabolism at 3 Years Postpartum. J Womens Health (Larchmt) 19, 941-950 (2010).

24. Pollitt, R. A., Rose, K. M. \& Kaufman, J. S. Evaluating the evidence for models of life course socioeconomic factors and cardiovascular outcomes: a systematic review. BMC Public Health 5, 1-13 (2005).

25. Murray, E. T. et al. Life course models of socioeconomic position and cardiovascular risk factors: 1946 birth cohort. Ann Epidemiol 21, 589-597 (2011).

26. Winkleby, M. A., Jatulis, D. E., Frank, E. \& Fortmann, S. P. Socioeconomic status and health: how education, income, and occupation contribute to risk factors for cardiovascular disease. Am J Public Health 82, 816-820 (1992).

27. Winkleby, M. A., Kraemer, H. C., Ahn, D. K. \& Varady, A. N. Ethnic and socioeconomic differences in cardiovascular disease risk factors: findings for women from the Third National Health and Nutrition Examination Survey, 1988-1994. JAMA 280, 356-362 (1998).

28. Schwarz, E. B. et al. Lactation and maternal risk of type 2 diabetes: a population-based study. Am J Med 123(863), e1-863e.6 (2010).

29. Stuebe, A. M., Rich-Edwards, J. W., Willett, W. C., Manson, J. E. \& Michels, K. B. Duration of lactation and incidence of type 2 diabetes. JAMA 294, 2601-2610 (2005).

30. Villegas, R. et al. Duration of breast-feeding and the incidence of type 2 diabetes mellitus in the Shanghai Women's Health Study. Diabetologia 51, 258-266 (2008).

31. Gunderson, E. P. et al. Lactation Duration and Midlife Atherosclerosis. Obstet Gynecol 126, 381-390 (2015).

32. McClure, C. K., Catov, J. M., Ness, R. B. \& Schwarz, E. B. Lactation and maternal subclinical cardiovascular disease among premenopausal women. Am J Obstet Gynecol 207, 46.e1-46.e8 (2012).

33. Schwarz, E. B. et al. Lactation and Maternal Measures of Subclinical Cardiovascular Disease. Obstet Gynecol 115, 41-48 (2010).

34. Cohen, S. S. et al. Parity and breastfeeding in relation to obesity among black and white women in the southern community cohort study. J Womens Health (Larchmt) 18, 1323-32 (2009).

35. Jiang, M. et al. Association between breastfeeding duration and postpartum weight retention of lactating mothers: A meta-analysis of cohort studies. Clin Nutr 37, 1224-1231 (2017).

36. Victora, C. G. \& Barros, F. C. Cohort Profile: The 1982 Pelotas (Brazil) Birth Cohort Study. Int J Epidemiol 35, 237-242 (2006).

37. Barros, F. C., Victora, C. G., Horta, B. L. \& Gigante, D. P. Methodology of the Pelotas birth cohort study from 1982 to 2004-5, Southern Brazil. Rev Saude Publica 42, 7-15 (2008).

38. Horta, B. L. et al. Cohort Profile Update: The 1982 Pelotas (Brazil) Birth Cohort Study. Int J Epidemiol 44, 441a-441e (2015).

39. Touboul, P. J. et al. Mannheim Carotid Intima- Media Thickness and Plaque Consensus (2004-2006-2011). Cerebrovasc Dis 34, 290-296 (2012).

40. Horta, B. L. et al. Early determinants of random blood glucose among adults of the 1982 birth cohort, Pelotas, Southern Brazil. Rev Saude Publica 42, 93-100 (2008).

41. Kehdy, F. S. et al. Origin and dynamics of admixture in Brazilians and its effect on the pattern of deleterious mutations. Proc Natl Acad Sci 112, 8696-8701 (2015).

42. Craig, C. L. et al. International physical activity questionnaire: 12-country reliability and validity. Med Sci Sports Exerc 35, 1381-1395 (2003)

\section{Acknowledgements}

This article is based on data from the study "Pelotas Birth Cohort, 1982" conducted by Postgraduate Program in Epidemiology at Universidade Federal de Pelotas with the collaboration of the Brazilian Public Health Association (ABRASCO). From 2004 to 2013, the Wellcome Trust supported the 1982 birth cohort study. The International Development Research Center, World Health Organization, Overseas Development Administration, European Union, National Support Program for Centers of Excellence (PRONEX), the Brazilian National Research Council $(\mathrm{CNPq})$, and the Brazilian Ministry of Health supported previous phases of the study. This study was financed in part by the Graduate Studies Coordinating Board (Capes; Finance Code 001). The funding organizations did not influence the study design, the data collection, the data analysis, the data interpretation, or the writing of the manuscript.

\section{Author Contributions}

N.P.L. collaborated in the data collection, conceived and performed the data analysis and wrote the manuscript. D.G.B. collaborated in the data analysis and in the writing of the manuscript. E.I.S.M. participated in the writing of the manuscript. F.C.B. designed the cohort study and collaborated in the writing of the manuscript. B.L.H. coordinated the 2012-13 follow-up visit of the cohort, conceived and planned the data analysis and collaborated in the writing of the manuscript. All authors reviewed the manuscript and approved the final version.

\section{Additional Information}

Supplementary information accompanies this paper at https://doi.org/10.1038/s41598-019-49576-1. 
Competing Interests: The authors declare no competing interests.

Publisher's note: Springer Nature remains neutral with regard to jurisdictional claims in published maps and institutional affiliations.

(c) (i) Open Access This article is licensed under a Creative Commons Attribution 4.0 International License, which permits use, sharing, adaptation, distribution and reproduction in any medium or format, as long as you give appropriate credit to the original author(s) and the source, provide a link to the Creative Commons license, and indicate if changes were made. The images or other third party material in this article are included in the article's Creative Commons license, unless indicated otherwise in a credit line to the material. If material is not included in the article's Creative Commons license and your intended use is not permitted by statutory regulation or exceeds the permitted use, you will need to obtain permission directly from the copyright holder. To view a copy of this license, visit http://creativecommons.org/licenses/by/4.0/.

(C) The Author(s) 2019 